\title{
Interpreting multiple dualities conjectured from superconformal index identities
}

\section{A. Khmelnitsky}

\author{
Department of Physics, CERN - Theory Division, \\ CH-1211 Geneva 23, Switzerland \\ Institute for Nuclear Research of the Russian Academy of Sciences, \\ 60th October Anniversary Prospect, 7a, 117312 Moscow, Russia \\ E-mail: khmeln@inr.ac.ru
}

\begin{abstract}
We consider field theory side of new multiple Seiberg dualities conjectured within superconformal index matching approach. We study the case of SU(2) supersymmetric QCD and find that the numerous conjectured duals are different faces of handful of master theories. These different faces are inequivalent to each other in a very peculiar sense. Some master theories are fully known; we construct superpotentials for others. We confirm that all index identities correspond to theories flowing to one and the same theory in the infrared, thus supporting the conjecture of index matching for Seiberg dual theories. However, none of the index identities considered in this paper actually implies an entirely new, unknown duality.
\end{abstract}

Keywords: Supersymmetry and Duality, Duality in Gauge Field Theories

ArXiv EPrint: 0912.4523 


\section{Contents}

1 Introduction and summary 1

$2 \quad N_{c}=2, N_{f}=4$ SQCD and its duals 3

3 Duality in the presence of accidental symmetry 5

4 Reduction to $N_{f}=3 \quad 8$

4.1 Flowing to the infrared 8

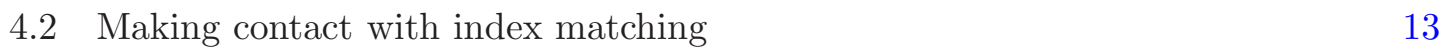

\section{Introduction and summary}

New method of exploring Seiberg dualities has been suggested recently. In refs. [1, 2], the generalisation of the Witten index for superconformal field theories was introduced, and it was conjectured that the indices of theories related by Seiberg duality should coincide [3]. The coincidence was checked for several known dual theories $[4,5]$. The index is a character of a relevant representation of certain subgroup of superconformal group and counts ground states invariant under the action of a particular supercharge. Thus, the conjecture seems natural to hold for models flowing to the same infrared conformal fixed point.

Superconformal indices for gauge theories are given in terms of elliptic hypergeometric integrals, and duality relations correspond to their highly non-trivial transformation properties [6-9]. Thus, the coincidence of indices is new independent argument in favour of duality conjecture. Comprehensive list of dualities and corresponding relations for elliptic hypergeometric integrals, as well as introduction to this recently emergent branch of special function theory and its relation to Seiberg dualities can be found in ref. [10].

On the other hand, known transformation properties of elliptic hypergeometric integrals lead to conjectures of new dualities between supersymmetric gauge theories [5, 10]. Although this method provides only field content of conjectured duals, it must be possible to construct complete field theories including their superpotentials. A remarkable feature of the superconformal index approach is that it suggests a multiplicity of duals to a single "electric" theory. By making use of this approach, Spiridonov and Vartanov [5] (SV in what follows) have recently conjectured 71 dual descriptions for supersymmetric QCD with $N_{c}=2$ colours and $N_{f}=4$ quark flavours whereas only three were known before that. For the SQCD with $N_{f}=3$ flavours, they have suggested 35 dual gauge theories. In the latter case, the low energy description in terms of non-gauge theory was found in ref. [11], but no non-trivial dual gauge theory was known.

In this paper we study the phenomenon of multiple duals. We consider duals conjectured by SV for $N_{c}=2, N_{f}=4,3 \mathrm{SQCD}$ and find that the relationship between theories 
behind the superconformal index identities can be called duality with reservations. There are two types of extra dualities. The first one is inherent in electric theories whose duals have enhanced accidental symmetries in the infrared (for the discussion of enhanced symmetries in the Seiberg duality context see, e.g., ref. [12]). Per se, these electric theories have only a few "master" duals. Dualities proliferate once some flavour current is coupled to external gauge field. Multiple dual theories then have the same field content, but differ by the structure of currents coupled to the external field.

Dualities of the second type are obtained from known ones by introducing a relevant operator into superpotential. This leads to new infrared behaviour of both electric and magnetic theories obtained by integrating out some heavy fields or after symmetry breaking. As the theories flow to the infrared, they can pass through intermediate stages; some of these intermediate theories are captured by the superconformal index approach. These theories are proper duals in the sense that they have the same infrared description, but usually they are not considered as new non-trivial independent dualities.

On the positive side, the superconformal index identities are in remarkable correspondence with field theory dualities understood in the above extended way. Field theories related by these identities do flow to the same infrared theory, and all index identities have their field theory counterparts. Thus, our study can be considered as a check of the conjecture that superconformal indices of Seiberg dual theories do match and contain important group-theoretical information on the structure of a theory.

The paper is organized as follows. In section 2 we summarize known dualities of $N_{c}=2$, $N_{f}=4$ SQCD. This theory has three "master" magnetic descriptions with different field contents and superpotentials [13-15]. Although the original electric theory has global symmetry group SU(8), two of its duals have lower symmetries. The latter symmetries get enhanced in the infrared, where the full SU(8) is restored [12]. Hence, part of SU(8) global symmetry is accidental in magnetic descriptions.

Since part of $\mathrm{SU}(8)$ is accidental in the two magnetic theories, there is an ambiguity in identifying the operators of the electric theory and their magnetic counterparts. We consider this point in section 3. The ambiguity becomes physical when the currents corresponding to Cartan generators of $\mathrm{SU}(8)$ are coupled to external gauge fields. Numerous magnetic theories in external gauge fields obtained in this way are inequivalent, and become identical in the infrared only. This construction is in one-to-one correspondence with SV counting based on superconformal index identities, hence giving the interpretation of multiple SV dualities in $N_{f}=4$ theory.

In section 4 we discuss $N_{f}=3$ theory as descendant of $N_{f}=4$. We will see explicitly that upon giving a mass to one electric quark flavour, all four $N_{f}=4$ theories (electric and three magnetic) flow to one and the same theory in the infrared. However, they do that through different intermediate steps. These steps depend on the way the quark mass term is introduced into the magnetic theory, so there are several intermediate theories. Some intermediate theories are precisely the duals suggested by SV via superconformal index approach. In this way, and with account for the phenomenon described in section 3, all SV duals are identified. Thus, we find that on the one hand, the superconformal index technique does produce valid results, and on the other hand, no entirely new non-trivial dualities are uncovered in this way. 


\begin{tabular}{|c|c|c|c|}
\hline Field content & Quark gauge invariants & Superpotential & Moduli \\
\hline $\begin{array}{l}Q^{i}(\mathbf{4}, \mathbf{1})^{+1} \\
\tilde{Q}_{\tilde{j}}(\mathbf{1}, \mathbf{4})^{-1}\end{array}$ & $\begin{array}{l}B^{i j} \equiv Q^{i} \cdot Q^{j}(\mathbf{6}, \mathbf{1})^{+2} \\
\tilde{B}_{\tilde{i} \tilde{j}} \equiv \tilde{Q}_{\tilde{i}} \cdot \tilde{Q}_{\tilde{j}}(\mathbf{1}, \mathbf{6})^{-2} \\
M_{\tilde{j}}^{i} \equiv Q^{i} \cdot \tilde{Q}_{\tilde{j}}(\mathbf{4}, \mathbf{4})^{0}\end{array}$ & & $\left(\begin{array}{rr}B^{i j} & M_{\tilde{j}}^{i} \\
-M_{\tilde{i}}^{j} & \tilde{B}_{\tilde{i} \tilde{j}}\end{array}\right)$ \\
\hline $\begin{array}{cc}q^{i} & (\mathbf{4}, \mathbf{1})^{-1} \\
\tilde{q}_{\tilde{j}} & (\mathbf{1}, \mathbf{4})^{+1} \\
B^{i j} & (\mathbf{6}, \mathbf{1})^{+2} \\
\tilde{B}_{\tilde{i} \tilde{j}} & (\mathbf{1}, \mathbf{6})^{-2} \\
\end{array}$ & $\begin{array}{l}C^{i j} \equiv q^{i} \cdot q^{j} \quad(\mathbf{6}, \mathbf{1})^{-2} \\
\tilde{C}_{\tilde{i} \tilde{j}} \equiv \tilde{q}_{\tilde{i}} \cdot \tilde{q}_{\tilde{j}} \\
N_{\tilde{j}}^{i} \equiv q^{i} \cdot \tilde{q}_{\tilde{j}}\end{array}$ & $\begin{array}{l}\frac{1}{4 \mu} \epsilon_{i j k l} B^{i j} q^{k} \cdot q^{l}+ \\
\frac{1}{4 \mu} \epsilon^{\tilde{i} \tilde{k} \tilde{k}} \tilde{B}_{\tilde{i} \tilde{j}} \tilde{q}_{\tilde{k}} \cdot \tilde{q}_{\tilde{l}}\end{array}$ & $\left(\begin{array}{rr}B^{i j} & N_{\tilde{j}}^{i} \\
-N_{\tilde{i}}^{j} & \tilde{B}_{\tilde{i} \tilde{j}}\end{array}\right)$ \\
\hline $\begin{array}{ll}q_{i} & (\overline{\mathbf{4}}, \mathbf{1})^{+1} \\
\tilde{q}^{j} & (\mathbf{1}, \overline{\mathbf{4}})^{-1} \\
M_{\tilde{j}}^{i} & (\mathbf{4}, \mathbf{4})^{0}\end{array}$ & $\begin{array}{l}C_{i j} \equiv q_{i} \cdot q_{j}(\overline{\mathbf{6}}, \mathbf{1})^{+2} \\
\tilde{C}^{\tilde{i} \tilde{j}} \equiv \tilde{q}^{\tilde{i}} \cdot \tilde{q}^{\tilde{j}} \quad(\mathbf{1}, \overline{\mathbf{6}})^{-2} \\
N_{i}^{\tilde{j}} \equiv q_{i} \cdot \tilde{q}^{\tilde{j}} \quad(\overline{\mathbf{4}}, \overline{\mathbf{4}})^{0}\end{array}$ & $\frac{1}{\mu} M_{\tilde{j}}^{i} q_{i} \cdot \tilde{q}^{\tilde{l}}$ & $\left(\begin{array}{rr}\epsilon^{i j k l} C_{k l} & M_{\tilde{j}}^{i} \\
-M_{\tilde{i}}^{j} & \epsilon_{\tilde{i} \tilde{j} \tilde{k} l} \tilde{C}^{k \hat{l} l}\end{array}\right)$ \\
\hline $\begin{array}{cl}q_{i} & (\overline{\mathbf{4}}, \mathbf{1})^{-1} \\
\tilde{q}^{j} & (\mathbf{1}, \overline{\mathbf{4}})^{+1} \\
B^{i j} & (\mathbf{6}, \mathbf{1})^{+2} \\
\tilde{B}_{\tilde{i} \tilde{j}} & (\mathbf{1}, \mathbf{6})^{-2} \\
M_{\tilde{j}}^{i} & (\mathbf{4}, \mathbf{4})^{0}\end{array}$ & $\begin{array}{l}C_{i j} \equiv q_{i} \cdot q_{j}(\overline{\mathbf{6}}, \mathbf{1})^{-2} \\
\tilde{C}^{\tilde{i j}} \equiv \tilde{q}^{\tilde{i}} \cdot \tilde{q}^{\tilde{j}} \quad(\mathbf{1}, \overline{\mathbf{6}})^{+2} \\
N_{i}^{\tilde{j}} \equiv q_{i} \cdot \tilde{q}^{\tilde{j}} \quad(\overline{\mathbf{4}}, \overline{\mathbf{4}})^{0}\end{array}$ & $\begin{array}{l}\frac{1}{\mu} M_{\tilde{j}}^{i} q_{i} \cdot \tilde{q}^{\tilde{l}}+ \\
\frac{1}{2 \mu} B^{i j} q_{i} \cdot q_{j}+ \\
\frac{1}{2 \mu} \tilde{B}_{\tilde{i} \tilde{j}} \tilde{q}^{i} \cdot \tilde{q}^{j}\end{array}$ & $\left(\begin{array}{rr}B^{i j} & M_{\tilde{j}}^{i} \\
-M_{\tilde{i}}^{j} & \tilde{B}_{\tilde{i} \tilde{j}}\end{array}\right)$ \\
\hline
\end{tabular}

Table 1. $N_{c}=2, N_{f}=4$ supersymmetric QCD and its duals.

\section{$2 \quad N_{c}=2, N_{f}=4 \mathrm{SQCD}$ and its duals}

Let us recall known properties of supersymmetric QCD with $\mathrm{SU}(2)$ gauge group and $N_{f}=4$ flavours of quarks, $Q^{i}$ in $\mathbf{2}$ representation and $\tilde{Q}_{\tilde{j}}$ in $\overline{\mathbf{2}}$ representation $(i, \tilde{j}=$ $1,2,3,4)$. Its feature is that fundamental and antifundamental representations of the gauge group are equivalent, so "left" quarks $Q$ and "right" quarks $\tilde{Q}$ are combined into one multiplet of $\mathrm{SU}(8)$ flavour group.

The theory is believed to have non-trivial infrared fixed point [13]. It has at least three Seiberg duals, i.e., theories which flow to the same fixed point in the infrared. All of them have $\mathrm{SU}(2)$ gauge group and at least $\mathrm{SU}(4)_{L} \times \mathrm{SU}(4)_{R} \times \mathrm{U}(1)_{B}$ global symmetry. They differ by field content, field representations and superpotentials. The pattern of the dual theories can be understood by considering their moduli spaces.

The moduli space is parametrized by all possible gauge invariants (modulo classical relations implied by their definitions) giving extremum to the superpotential. In original electric theory, there is no superpotential and moduli space is spanned by expectation values of gauge invariants constructed from quarks. These are mesons $M_{\tilde{j}}^{i}=Q^{i} \cdot \tilde{Q}_{\tilde{j}}$, baryons $B^{i j}=Q^{i} \cdot Q^{j}$ and antibaryons $\tilde{B}_{\tilde{i} \tilde{j}}=\tilde{Q}_{\tilde{i}} \cdot \tilde{Q}_{\tilde{j}}$. Because of enhanced flavour symmetry, they form together antisymmetric tensor representation of $\mathrm{SU}(8)$. Thus, all other descriptions of this infrared fixed point must have the same moduli space. In case of smaller flavour group, additional symmetry should accidentally emerge in the infrared.

Electric SQCD and all three duals are described in table 1, with the representations of the common global symmetry group $\mathrm{SU}(4)_{L} \times \mathrm{SU}(4)_{R} \times \mathrm{U}(1)_{B}$. The original theory 
and the third (last) dual possess $\mathrm{SU}(8)$ flavour symmetry. For the first two duals, it is impossible to arrange elementary fields in the SU(8) multiplets, and flavour SU(8) emerges accidentally in the infrared.

The tree duals are constructed by introducing to SQCD some of the moduli of electric theory as elementary fields. Then one has to get rid of similar composite moduli in order to restore the proper moduli space structure. To this end, a superpotential is introduced. Representations of magnetic quarks $q, \tilde{q}$ are then completely fixed by demanding the coincidence of moduli spaces. In order to match elementary scalars to electric composites of canonical dimension 2, a new energy scale $\mu$ is introduced.

The first dual description in our table was considered by Csáki et al. [14] and contains baryons $B^{i j}$ and antibaryons $\tilde{B}_{\tilde{i} \tilde{j}}$ of electric QCD as elementary fields. The presence of these fields explicitly breaks $\mathrm{SU}(8)$ symmetry down to $\mathrm{SU}(4)_{L} \times \mathrm{SU}(4)_{R} \times \mathrm{U}(1)_{B}$. There is the superpotential, which gives the mass to composite magnetic baryons $C^{i j} \equiv q^{i} \cdot q^{j}$ and antibaryons $\tilde{C}_{\tilde{i} \tilde{j}} \equiv \tilde{q}_{\tilde{i}} \cdot \tilde{q}_{\tilde{j}}$. This superpotential is crucial to match moduli spaces. The moduli space of this dual theory is parametrized by $\mathrm{SU}(8)$ antisymmetric tensor containing elementary baryons and composite mesons ${ }^{1} N_{\tilde{j}}^{i} \equiv q^{i} \cdot \tilde{q}_{\tilde{j}}$. With this identification of moduli spaces, one relates electric gauge invariants to magnetic ones surviving in the infrared. Thus, one identifies baryons $B^{i j}$ and antibaryons $\tilde{B}_{\tilde{i} \tilde{j}}$ present in both theories, and mesons $M_{\tilde{j}}^{i}$ of electric theory with magnetic ones $N_{\tilde{j}}^{i}$. Composite baryons $C^{i j}, \tilde{C}_{\tilde{i} \tilde{j}}$ are absent in the infrared because of the superpotential; they do not have electric counterparts.

The generalisation of this duality to higher rank gauge groups has been found recently by using superconformal index identities ( $S U-S P$ series of [10]).

The second dual was considered in the original paper by Seiberg [13] as part of the series of dual theories with $\mathrm{SU}\left(N_{c}\right)$ gauge groups. Electric mesons $M_{\tilde{j}}^{i}$ are introduced as elementary fields and, together with composite baryons $C_{i j} \equiv q_{i} \cdot q_{j}$ and antibaryons $\tilde{C}^{\tilde{i} \tilde{j}} \equiv \tilde{q} \tilde{q}^{\tilde{i}} \cdot \tilde{q}^{\tilde{j}}$, they parametrize the moduli space. Superpotential takes care of now redundant composite mesons $N_{i}^{\tilde{j}} \equiv q_{i} \cdot \tilde{q}^{\tilde{j}}$. One identifies mesons $M_{\tilde{j}}^{i}$ of electric and dual theories, and electric baryons $B^{i j}, \tilde{B}_{\tilde{i} \tilde{j}}$ with the Hodge-duals of magnetic baryons $\epsilon^{i j k l} C_{k l}$ and $\epsilon_{\tilde{i} \tilde{j} \tilde{k} \tilde{l}} \tilde{C}^{\tilde{k} \tilde{l}}$. Similarly to the first dual, the existence of elementary mesons explicitly breaks $\mathrm{SU}(8)$ symmetry down to $\mathrm{SU}(4)_{L} \times \mathrm{SU}(4)_{R} \times \mathrm{U}(1)_{B}$.

The third dual theory was proposed by Intriligator and Pouliot in [15], where they generalise the original Seiberg series of dual theories to $S P\left(N_{c}\right)$ gauge groups. The full set of electric colour singlets is added as fundamental fields, and all composite singlets are made massive by the superpotential. Thus, the moduli space is trivially the same as electric one, and all electric composites are identified with corresponding elementary fields in the dual theory. As the full set of SQCD gauge invariants fits in SU(8) multiplet, this dual has SU(8) global symmetry and does not exhibit accidental symmetry in the infrared.

\footnotetext{
${ }^{1}$ In electric theory, there are classical relations between mesons and baryons. In SU(8) language, they state that the rank of the antisymmetric matrix parameterizing the moduli space does not exceed 2 . In magnetic theory, the rank of the moduli matrix is constrained partly by the superpotential and partly by the fact that baryon vev of rank greater than 2 leads to runaway vacuum.
} 


\section{Duality in the presence of accidental symmetry}

The first and second duals of the previous section possess only $\mathrm{SU}(4)_{L} \times \mathrm{SU}(4)_{R} \times \mathrm{U}(1)_{B}$ global symmetry, which gets promoted in the infrared to the full $\mathrm{SU}(8)$ of the electric theory. This is not in contradiction with the concept of Seiberg duality which relates different theories with the same infrared properties. The dual theories may have not only different gauge groups and field contents, but also different global symmetries.

It is worth mentioning that the 't Hooft anomaly matching conditions [16] are somewhat peculiar in this situation. As the flavour group of the magnetic theory is smaller than that of its infrared descendant, anomaly matching conditions in the magnetic theory apply to smaller set of global currents. In other words, not all anomaly relations of the electric theory have their magnetic counterparts, simply because the magnetic theory has smaller set of global currents.

Once the global groups of electric theory and its magnetic dual are different, there is an ambiguity in identifying the operators of the two theories. Operators related by global symmetry in electric theory may no longer have this property in magnetic theory; this ambiguity becomes irrelevant in the infrared only. Hence, a small deformation of the electric theory may have several duals emanating from one and the same undeformed magnetic master theory. As an example, as proposed in ref. [12], one can modify electric and magnetic theories by introducing small terms into their superpotentials. These terms are related by duality correspondence, and it is expected that duality remains valid for modified theories. Above the infrared, this correspondence is ambiguous, and unique superpotential term in theory with larger symmetry corresponds to a family of inequivalent terms in dual theory.

Consider small mass term for a pair of quarks in electric theory of the previous section. For definiteness, take it as $m Q^{4} \cdot \tilde{Q}_{\tilde{4}}$. Its magnetic counterpart in the first dual theory, obtained according to the above default identification, is $m q^{4} \cdot \tilde{q}_{\tilde{4}}$ (up to a constant factor, see eq. (4.3)). However, the $\mathrm{SU}(8)$ rotation of the mass term, innocent in electric theory, gives rise to terms $m B^{34}, m \tilde{B}_{\tilde{3} \tilde{4}}$ in the superpotential of the magnetic theory, as well as their linear combinations with $m q^{4} \cdot \tilde{q}_{\tilde{4}}$. These cannot be rotated back to $m q^{4} \cdot \tilde{q}_{\tilde{4}}$ by $\mathrm{SU}(4) \times \mathrm{SU}(4) \times \mathrm{U}(1)$ of the "magnetic theory". In the infrared, $q^{4} \cdot \tilde{q}_{4}$ is replaced by magnetic meson $N_{\tilde{4}}^{4}$, and all mass terms in the dual theory are related by the accidental $\mathrm{SU}(8)$.

Likewise, the modifications of the second dual theory in addition to default magnetic counterpart $m M_{\tilde{4}}^{4}$ contain also superpotential terms $m q_{1} \cdot q_{2}, m \tilde{q}^{\tilde{1}} \cdot \tilde{q}^{\tilde{2}}$ and their linear combinations with $m M_{\tilde{4}}^{4}$. These cannot be related to each other by $\mathrm{SU}(4) \times \mathrm{SU}(4) \times$ $\mathrm{U}(1)$ transformations.

We see that once introduced, the mass term leads to a family of inequivalent dual theories emanating from one master theory. In this sense, $N_{c}=2, N_{f}=4 \mathrm{SQCD}$ with small mass term for quark flavour has two continuous families of dual descriptions, namely, the first and second magnetic duals with inequivalent families of terms in the superpotentials.

Our point is that it is this kind of multiplicity that has been found by SV using superconformal index matching. For $N_{c}=2, N_{f}=4 \mathrm{SQCD}$, the matching approach suggests that there are 72 theories dual to each other. These include the original electric 
theory, the third dual from our table and two sets of 35 dual theories corresponding to the first and second magnetic duals. Duals in each set have identical field content and Lagrangian, namely, those of the first or second magnetic theories. The only difference between them is the way the anomalies match with electric theory, i.e., the way $\mathrm{SU}(4)_{L} \times$ $\mathrm{SU}(4)_{R} \times \mathrm{U}(1)_{B}$ is embedded into $\mathrm{SU}(8)$. This part of multiplicity is precisely due to the ambiguity in relating operators in dual theories.

As they stand, all 35 duals are equivalent to their master theory. To make this multiplicity physical, one deforms the electric theory. Instead of using $\mathrm{SU}(8)$ breaking terms in superpotential as proposed in [12], we introduce external gauge field that weakly couples to global current of the Cartan subgroup of SU(8). Then the family of dualities found by $\mathrm{SV}$ in the superconformal index context are dualities between the theories in the external field coupled to inequivalent global currents.

To proceed further, we note that $\mathrm{SU}(4)_{L} \times \mathrm{SU}(4)_{R} \times \mathrm{U}(1)_{B}$ of the first and second magnetic theories is embedded in electric $\mathrm{SU}(8)$ in non-trivial way.

Consider SU(8) Cartan generator represented on electric quarks by $\operatorname{diag}\left(x_{i}, \tilde{x}_{j}\right)$ with $i, j=1,2,3,4$. Let us find its representation on magnetic quarks using the identification of gauge invariants. Suppose that this operator is represented on magnetic quarks and elementary baryons of the first dual theory by $\operatorname{diag}\left(z_{i}, \tilde{z}_{j}\right)$ and $\operatorname{diag}\left(y_{i}, \tilde{y}_{j}\right)$, respectively. Demanding that the elementary magnetic baryons have the same charges as composite electric ones, we obtain $x_{i}=y_{i}$ and $\tilde{x}_{j}=\tilde{y}_{j}$. Invariance of the superpotential terms $\epsilon_{i j k l} B^{i j} q^{k} \cdot q^{l}$ and $\epsilon^{\tilde{i} \tilde{j} \tilde{k} \tilde{l}} \tilde{B}_{\tilde{i} \tilde{j}} \tilde{q}_{\tilde{k}} \cdot \tilde{q}_{\tilde{l}}$ (see table 1) gives for the charges of the magnetic quarks

$$
\begin{aligned}
& z_{i}=y_{i}-\frac{1}{2} \sum_{k} y_{k}=x_{i}-\frac{1}{2} \sum_{k} x_{k}, \\
& \tilde{z}_{j}=\tilde{y}_{j}-\frac{1}{2} \sum_{l} \tilde{y}_{l}=\tilde{x}_{j}-\frac{1}{2} \sum_{l} \tilde{x}_{l} .
\end{aligned}
$$

Charges of magnetic quarks of the second dual theory with elementary mesons are obtained in a similar way,

$$
\begin{aligned}
& z_{i}=-x_{i}+\frac{1}{2} \sum_{k} x_{k}, \\
& \tilde{z}_{j}=-\tilde{x}_{j}+\frac{1}{2} \sum_{l} \tilde{x}_{l} .
\end{aligned}
$$

Remarkably, these relationships were found by SV as transformations acting on arguments of superconformal indices that lead to the duality identities. The arguments of index are precisely the global group elements, parametrized by eigenvalues of their matrix representation. It turns out that these transformations together with permutations of quarks generate the Weyl group $W\left(E_{7}\right)$ of the exceptional root system $E_{7}$, which defines transformational properties of hyperelliptic integrals that give indices of dual theories.

Consider now baryon charge represented on electric quarks by

$$
Q_{B}=\operatorname{diag}(+1,+1,+1,+1,-1,-1,-1,-1) .
$$


With the above identifications, we see that magnetic quarks of the first (second) dual theory have the opposite (same) baryon charges as electric ones, in accord with table 1. There are two other representations of baryon charge on electric quarks that lead to inequivalent representations on magnetic quarks. We choose them in electric theory as follows,

$$
\begin{aligned}
& Q_{B}^{\prime}=\operatorname{diag}(+1,+1,+1,-1,-1,-1,-1,+1), \\
& Q_{B}^{\prime \prime}=\operatorname{diag}(+1,+1,-1,-1,-1,-1,+1,+1) .
\end{aligned}
$$

The corresponding representations on magnetic quarks are

$$
\begin{aligned}
& Q_{B 1}^{\prime}=\operatorname{diag}(0,0,0,-2,0,0,0,+2), \\
& Q_{B 1}^{\prime \prime}=\operatorname{diag}(+1,+1,-1,-1,-1,-1,+1,+1)
\end{aligned}
$$

for the first dual and

$$
\begin{aligned}
& Q_{B 2}^{\prime}=\operatorname{diag}(0,0,0,+2,0,0,0,-2), \\
& Q_{B 2}^{\prime \prime}=\operatorname{diag}(-1,-1,+1,+1,+1,+1,-1,-1)
\end{aligned}
$$

for the second dual.

Coupling of external field to the baryon current breaks SU(8) symmetry of electric theory down to $\mathrm{SU}(4) \times \mathrm{SU}(4) \times \mathrm{U}(1)_{B}$. The pattern of symmetry breaking in magnetic theories depends on the representation of baryon charge. Coupling of the external field to the charge $Q_{B}$ does not break $\mathrm{SU}(4) \times \mathrm{SU}(4) \times \mathrm{U}(1)_{B}$ symmetry at all. Coupling to $Q_{B}^{\prime}$ and $Q_{B}^{\prime \prime}$ breaks each of $\mathrm{SU}(4)$ down to $\mathrm{SU}(3) \times \mathrm{U}(1)$ and $\mathrm{SU}(2) \times \mathrm{SU}(2) \times \mathrm{U}(1)$, respectively. Hence, the resulting magnetic theories in external field are physically different. The fact that anomalies of weird baryon charges $Q_{B}^{\prime}$ and $Q_{B}^{\prime \prime}$ match to those of electric baryon current gives extra evidence that magnetic theory possesses the full $\mathrm{SU}(8)$ symmetry in the infrared.

Thus, upon coupling different representations of the baryon current to external field, each of the two duals split into three inequivalent theories. These are the Seiberg duals to electric SQCD in external field coupled to the baryon current. Note that one of the above dualities is precisely the explicit example given by SV in tables 5,6 of [5]; the fields are split into representations of

$$
\mathrm{SU}(3)_{L} \times \mathrm{U}(1)_{L} \times \mathrm{SU}(3)_{R} \times \mathrm{U}(1)_{R} \times \mathrm{U}(1)_{B}
$$

and $\mathrm{U}(1)_{B}$ charges coincide with our $Q_{B}^{\prime}$. Electric counterparts of the $\mathrm{U}(1)_{L / R}$ charges are given by

$$
\begin{aligned}
& Q_{L}=\operatorname{diag}(-1,-1,-1,0,0,0,0,3) \\
& Q_{R}=\operatorname{diag}(0,0,0,3,-1,-1,-1,0) .
\end{aligned}
$$

In magnetic theories, these are precisely the $\mathrm{U}(1)$-subgroups remaining after $\mathrm{SU}(4)_{L / R}$ split into $\mathrm{SU}(3) \times \mathrm{U}(1)$ in external field interacting with the charge $Q_{B}^{\prime}$. 


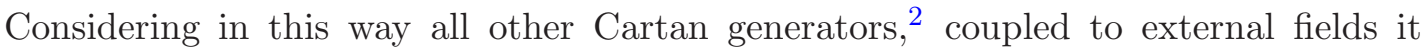
is straightforward to obtain the two complete sets of 35 dual theories suggested by SV. They correspond to $\frac{1}{2} C_{8}^{4}$ splittings of eight eigenvalues into two groups acting on left and right magnetic quarks, respectively, modulo interchanging the notions of left and right. Remarkably, there is one-to-one correspondence between this procedure and the index approach: no extra dualities are obtained in our field-theoretic way as compared to SV.

We see that multiplicity of dual descriptions suggested by superconformal index matching reveals some new aspects of duality. We have found that most of 72 dual theories suggested by superconformal index matching can be considered independent only in quite unconventional sense, namely, by coupling different global currents of the same theory to external field. Without this additional construction, there are only four theories dual to each other. It is worth noting, though, that several aspects of multiple duality, like the rôle of $W\left(E_{7}\right)$ transformations that somehow relate all four duals, remain unclear.

\section{Reduction to $N_{f}=3$}

One way of studying known dualities and obtaining new ones is to consider the flow of dual theories under integration out some of the fields. SV proposed a reduction procedure for elliptic hypergeometric integral identities that starts with an established relation between superconformal indices of two theories and gives a relation for theories with lower rank of global symmetry group. One expects that this reduction corresponds to integration out some of the matter fields in pertinent field theories. SV considered the reduction that effectively removes one quark flavour from $N_{c}=2, N_{f}=4 \mathrm{SQCD}$ and its duals. As a result, a set of 36 gauge theories dual to each other was proposed. Only tree of them have different field content. One of these theories is $N_{f}=3, N_{c}=2$ SQCD without extra fields, while two others are $N_{f}=3, N_{c}=2 \mathrm{SQCD}$ with two different sets of additional gauge singlets.

At the same time, it is known that electric and the second and third dual theories flow to the same theory in the infrared. The low energy theory does not have gauge symmetry and is a theory of one $\mathrm{SU}(6)$ antisymmetric tensor field $V$ with superpotential proportional to Pfaffian of $V$ [11].

These two views on the result of integrating out one quark flavor from $N_{c}=2, N_{f}=4$ SQCD are in apparent contradiction. To see what happens, let us systematically study how the theories listed in table 1 flow towards the infrared. Of particular interest are the theories that emerge as intermediate steps: we will see in section 4.2 that some of them are precisely the duals suggested by the index matching approach.

\subsection{Flowing to the infrared}

Let us leave aside for the time being the conjectures based on superconformal index approach, and integrate out one quark flavor and its magnetic counterparts in all four theories described in section 2. For the second and the third dual descriptions this procedure was

\footnotetext{
${ }^{2}$ In fact, it is sufficient to consider the least symmetric Cartan generators that break $\mathrm{SU}(8)$ down to $[\mathrm{U}(1)]^{7}$.
} 
briefly described in the original papers $[13,15]$. Here we follow the work [12] where identifications of operators in electric and magnetic theories were discussed.

We are going to add either the mass term for the fourth flavor, $m Q^{4} \cdot \tilde{Q}_{\tilde{4}}$, or oblique mass term $h Q^{3} \cdot Q^{4}$. Although these terms are related by SU(8) transformation in electric theory, they have different counterparts in the first dual theory with the default identification of the operators. This has been discussed in section 3. Accordingly, the flow of the first dual theory to the infrared proceeds through different steps for the two mass terms. The same applies to the second dual theory. One can see that our choice of the mass terms exhausts all possible patterns of flow; considering other mass terms adds nothing new.

We discuss electric theory first. Keeping explicit $\mathrm{SU}(4)_{L} \times \mathrm{SU}(4)_{R} \times \mathrm{U}(1)_{B}$ part of $\mathrm{SU}(8)$ global symmetry, we begin with the mass term $m Q^{4} \cdot \tilde{Q}_{\tilde{4}}$. For $m \gg \Lambda$, the dynamical scale of electric theory, massive quarks are integrated out, and one arrives at SQCD with $N_{f}=3$ flavours. Below its own dynamical scale $\Lambda_{3}=\left(m \Lambda^{2}\right)^{1 / 3}$ this theory confines and generates dynamical superpotential [11]

$$
W_{\mathrm{dyn}}=\frac{1}{m \Lambda^{2}}\left\{\frac{1}{4} \epsilon_{r s t} B^{r s} M_{\tilde{t}}^{t} \tilde{B}_{\tilde{r} \tilde{s}} \epsilon^{\tilde{r} \tilde{s} \tilde{t}}-\operatorname{det} M\right\}=\frac{\operatorname{Pf} V}{\Lambda_{3}^{3}} .
$$

Here $r, \tilde{r}, \ldots=1,2,3$ are the indices of $\mathrm{SU}(3)_{L} \times \mathrm{SU}(3)_{R}$ flavour group that remains after integration out. The actual full global symmetry is $\mathrm{SU}(6)$, and $V$ is antisymmetric $\mathrm{SU}(6)$ tensor composed of mesons and baryons.

Let us now consider oblique mass term $h Q^{3} \cdot Q^{4}$. It breaks flavour group in another manner: $\mathrm{SU}(4)_{L} \times \mathrm{SU}(4)_{R} \times \mathrm{U}(1)_{B} \subset \mathrm{SU}(8) \rightarrow \mathrm{SU}(2)_{L} \times \mathrm{SU}(4)_{R} \times \mathrm{U}(1) \subset \mathrm{SU}(6)$. Integrating out massive quarks, we arrive at SQCD with two surviving left quarks $Q^{a}, a=1,2$ and four right quarks $\tilde{Q}_{\tilde{j}}$. This is still $N_{f}=3$ SQCD. Below the scale $\left(h \Lambda^{2}\right)^{1 / 3}$ quarks are confined in mesons $M_{\tilde{j}}^{a}$, baryons $B^{a b}$ and antibaryons $\tilde{B}_{\tilde{i} \tilde{j}}$. Dynamically generated superpotential is given by eq. (4.1) with $\tilde{Q}_{\tilde{4}}$ substituted for $Q^{3}$ :

$$
W_{\mathrm{dyn}}=\frac{1}{4 h \Lambda^{2}}\left\{M_{\tilde{i}}^{a} M_{\tilde{j}}^{b}-\frac{1}{4} B^{a b} \tilde{B}_{\tilde{i} \tilde{j}}\right\} \epsilon^{\tilde{i} \tilde{j} \tilde{k} \tilde{l}} \epsilon_{a b} \tilde{B}_{\tilde{k} \tilde{l}} .
$$

This description also possesses the full $\mathrm{SU}(6)$ global symmetry. It is equivalent to the previous one after field redefinition, as should be the case since the two low energy descriptions originate from one and the same theory with mass terms related by $\mathrm{SU}(8)$ transformation.

Thus, the electric theory flows through $N_{f}=3$ SQCD with no gauge singlet fields, whose low energy description is the theory of $\mathrm{SU}(6)$ antisymmetric tensor field.

Let us consider what happens with the first dual theory as we add each of the mass terms. Before writing counterparts of the mass terms we note one subtlety in the operator identification. While electric composite baryons and antibaryons match the corresponding elementary magnetic fields directly ${ }^{3}$ (see table 1), composite mesons match up to nontrivial factor:

$$
M_{\tilde{j}}^{i} \simeq \sqrt{\frac{-\Lambda^{2}}{\mu^{2}}} N_{\tilde{j}}^{i} \simeq \sqrt{\frac{-\Lambda^{2}}{\mu^{2}}} q^{i} \cdot \tilde{q}_{\tilde{j}},
$$

\footnotetext{
${ }^{3}$ Because of different canonical dimensions of these fields, this matching involves a scale $\mu$.
} 
where the scale $\mu$ is introduced on dimensional grounds. This identification ensures that the duality transformation applied twice gives back the original theory.

With this qualification, the electric theory with mass term $m Q^{4} \cdot \tilde{Q}_{\tilde{4}}$ is dual to the the magnetic one with the following superpotential,

$$
W=\frac{1}{4 \mu} \epsilon_{i j k l} B^{i j} q^{k} \cdot q^{l}+\frac{1}{4 \mu} \epsilon^{\tilde{i} \tilde{j} \tilde{k} \tilde{l}} \tilde{B}_{\tilde{i} \tilde{j}} \tilde{q}_{\tilde{k}} \cdot \tilde{q}_{\tilde{l}}+m \sqrt{\frac{-\Lambda^{2}}{\mu^{2}}} q^{4} \cdot \tilde{q}_{\tilde{4}} \cdot
$$

For large $m$, quarks $q^{4}$ and $\tilde{q}_{\tilde{4}}$ are heavy. Integrating them out we arrive at the theory with $N_{f}=3$ quark flavours and the same set of elementary baryons as in the original magnetic $N_{f}=4$ theory. The tree level superpotential after integration out is given by

$$
W_{\text {tree }}=\frac{1}{2 \mu} \epsilon_{r s t} q^{r} \cdot q^{s} B^{t 4}+\frac{1}{2 \mu} \epsilon^{\tilde{r} \tilde{s} \tilde{t}} \tilde{q}_{\tilde{r}} \cdot \tilde{q}_{\tilde{s}} \tilde{B}_{\tilde{t} \tilde{4}}-\frac{1}{4 m \mu^{2}} \sqrt{\frac{\mu^{2}}{-\Lambda^{2}}} \epsilon_{r s t} \epsilon^{\tilde{r} \tilde{s} \tilde{t}} B^{r s} \tilde{B}_{\tilde{r} \tilde{s}} q^{t} \cdot \tilde{q}_{\tilde{t}},
$$

where all indices take the values $1,2,3$. This theory has its own scale given by

$$
\tilde{\Lambda}_{3} \equiv\left(m \frac{\Lambda}{\mu} \tilde{\Lambda}^{2}\right)^{1 / 3}=(m \tilde{\Lambda} \mu)^{1 / 3}
$$

where $\tilde{\Lambda} \equiv \mu^{2} / \Lambda$ is the scale of the initial magnetic theory. Below this scale the theory confines and generates dynamical superpotential analogous to (4.1):

$$
W_{\mathrm{dyn}}=\frac{1}{m \tilde{\Lambda}^{2}} \sqrt{\frac{\mu^{2}}{-\Lambda^{2}}}\left\{\frac{1}{4} \epsilon_{r s t} C^{r s} N_{\tilde{t}}^{t} \tilde{C}_{\tilde{r} \tilde{s}} \epsilon^{\tilde{r} \tilde{s} \tilde{t}}-\operatorname{det} N\right\}
$$

where $C^{r s} \equiv q^{r} \cdot q^{s}$ and $\tilde{C}_{\tilde{r} \tilde{s}} \equiv \tilde{q}_{\tilde{r}} \cdot \tilde{q}_{\tilde{s}}$ are composite magnetic baryons and antibaryons of $N_{f}=3$ theory.

Upon rescaling the meson field $N_{\tilde{j}}^{i}$ to match the electric one $M_{\tilde{j}}^{i}$, we obtain the theory of interacting mesons and baryons (the latter are $B^{r s}, B^{r 4}, C^{r s}$ and their antibaryons) with the superpotential given by the sum of $W_{\text {dyn }}$ and $W_{\text {tree }}$ :

$$
\begin{aligned}
& W=\frac{1}{m \Lambda^{2}}\left\{\frac{1}{4} \epsilon_{r s t} B^{r s} M_{\tilde{t}}^{t} \tilde{B}_{\tilde{r} \tilde{s} \tilde{\epsilon}} \epsilon^{\tilde{r} \tilde{t}}-\operatorname{det} M\right\}+ \\
& \quad \frac{1}{2 \mu} \epsilon_{r s t} C^{r s} B^{t 4}+\frac{1}{2 \mu} \epsilon^{\tilde{r} \tilde{s} \tilde{t}} \tilde{C}_{\tilde{r} \tilde{s}} \tilde{B}_{\tilde{t} \tilde{4}}-\frac{1}{4 m \mu^{2}} \epsilon_{r s t} C^{r s} M_{\tilde{t}}^{t} \tilde{C}_{\tilde{r} \tilde{s}} \epsilon^{\tilde{r} \tilde{s} \tilde{t}} .
\end{aligned}
$$

According to our definition of $B$, the canonically normalized field is $B / \mu$. Since $C$ is the composite field arising because of strong coupling at $\tilde{\Lambda}_{3}$, its canonically normalized form is $C / \tilde{\Lambda}_{3} \simeq q \cdot q / \tilde{\Lambda}_{3}$. Hence, this superpotential gives masses of the same scale $\tilde{\Lambda}_{3}$ to all baryons absent in electric $N_{f}=3$ theory. Integrating them out we are left with the same theory as in the electric case with the superpotential given by the first line of eq. (4.5), cf. eq. (4.1).

Hence, the electric theory and its first dual flow to the same theory of interacting singlets. Notably, the flow of the first dual proceeds through the intermediate description. This is $N_{f}=3 \mathrm{SQCD}$ with six baryons $B^{r s}, B^{r 4}$, six antibaryons $\tilde{B}_{\tilde{r} \tilde{s}}, \tilde{B}_{\tilde{s} \tilde{4}}$ and superpotential given by eq. (4.4). The flavour group of this theory is

$$
\mathrm{SU}(3)_{L} \times \mathrm{SU}(3)_{R} \times \mathrm{U}(1)_{B} \times \mathrm{U}(1)_{4},
$$


where the last factor is inherited from the initial magnetic theory together with extra baryons $B^{r 4}, \tilde{B}_{\tilde{s} \tilde{4}}$. In electric theory, this symmetry acts only on massive quarks and disappears once these quarks have been integrated out.

Let us now turn to the second electric mass term $h Q^{3} \cdot Q^{4}$. It corresponds to the term $h B^{34}$ in the first magnetic dual. The full superpotential is now given by

$$
W=\frac{1}{4 \mu} \epsilon_{i j k l} B^{i j} q^{k} \cdot q^{l}+h B^{34}+\frac{1}{4 \mu} \epsilon^{\tilde{\epsilon} \tilde{j} \tilde{k} \tilde{l}} \tilde{B}_{\tilde{i} \tilde{j}} \tilde{q}_{\tilde{k}} \cdot \tilde{q}_{\tilde{l}} \cdot
$$

It generates non-zero expectation value for magnetic quarks,

$$
q^{1} \cdot q^{2}=-\mu h \equiv v^{2},
$$

which completely breaks the gauge group. Let us choose the quark vev to be $\left(q^{a}\right)_{\alpha}=v \delta_{\alpha}^{a}$, where $\alpha, \ldots=1,2$ is colour index. Then for $h \gg \tilde{\Lambda}$, the fields $\left(q^{a}\right)_{a}$ as well as $\left(q^{3}\right)_{\alpha}$, $\left(q^{4}\right)_{\alpha}$ and baryons, except for $B^{12}$, obtain masses and are integrated out. The fields $\left(q^{1}\right)_{2}$, $\left(q^{2}\right)_{1}$ and $B^{12}$ remain massless but completely decouple. Thus we are left with eight quark components $\left(\tilde{q}_{\tilde{j}}\right)_{\alpha}$ and antibaryons $\tilde{B}_{\tilde{i} \tilde{j}}$ with superpotential given by the last term in eq. (4.7).

Using the quark vev we rescale quark fields to match them to electric mesons:

$$
\left(\tilde{q}_{\tilde{i}}\right)_{\alpha} \equiv-\epsilon_{\alpha b} \frac{1}{v} \sqrt{\frac{\mu^{2}}{-\Lambda^{2}}} M_{\tilde{i}}^{b} .
$$

Tree level superpotential now reads

$$
W_{\text {tree }}=\frac{1}{4 h \Lambda^{2}} \epsilon_{a b} M_{\tilde{i}}^{a} M_{\tilde{j}}^{b} \tilde{B}_{\tilde{k} \tilde{l}} \epsilon^{\tilde{i} \tilde{k} \tilde{k} \tilde{l}} .
$$

It matches the first term in eq. (4.2).

The remaining part of superpotential is generated dynamically. To check this, one gives large vevs to all baryon and antibaryon fields except for $B^{34}$. Due to superpotential (4.7), baryon vevs provide masses to all quarks except for $q^{1}$ and $q^{2}$. Integrating the massive quarks out one obtains a theory with one quark flavour composed of $q^{1}$ and $q^{2}$ and unbroken $\mathrm{SU}(2)$ colour group. This theory confines and generates non-perturbative dynamical superpotential [11]

$$
W_{\mathrm{dyn}}=\frac{\left(\tilde{\Lambda}_{1}\right)^{5}}{\left\langle q^{1} \cdot q^{2}\right\rangle}
$$

Here $\tilde{\Lambda}_{1}$ is the scale of the resulting $N_{f}=1$ theory. It is determined by baryon vevs and the scale of original theory,

$$
\left(\tilde{\Lambda}_{1}\right)^{5}=\operatorname{Pf}\left[B^{a b} \oplus \tilde{B}_{\tilde{i} \tilde{j}}\right] \mu^{-3} \tilde{\Lambda}^{2}=\frac{\mu}{16 \Lambda^{2}} \epsilon_{a b} B^{a b} \epsilon^{\tilde{j} \tilde{j} \tilde{k} \tilde{l}} \tilde{B}_{\tilde{i} \tilde{j}} \tilde{B}_{\tilde{k} \tilde{l}},
$$

where we have used the relation $\Lambda \tilde{\Lambda}=\mu^{2}$ between the scales of electric and magnetic theories. Using this expression and the quark vev (4.8), one obtains the dynamically generated superpotential

$$
W_{\mathrm{dyn}}=-\frac{1}{16 h \Lambda^{2}} \epsilon_{a b} B^{a b} \epsilon^{\tilde{i} \tilde{j} \tilde{k} \tilde{l}} \tilde{B}_{\tilde{i} \tilde{j}} \tilde{B}_{\tilde{k} \tilde{l}}
$$


which is precisely the second term in eq. (4.2). In this way we arrive at the same theory of the antisymmetric SU(6) tensor field we have obtained starting from electric theory. As in the previous case, part of the superpotential is given by tree-level terms while the other part is generated non-perturbatively. As opposed to the previous case, however, the term $h B^{34}$ breaks the gauge group and the theory does not pass through any intermediate description during the flow.

Now let us turn to the second dual theory. Adding the electric mass term $m Q^{4} \cdot \tilde{Q}_{\tilde{4}}$ induces the term $m M_{\tilde{4}}^{4}$ in this magnetic dual. The resulting flow resembles closely that of the first dual with the term $h B^{34}$. As described by Seiberg [13], the term $m M_{\tilde{4}}^{4}$ in superpotential leads to non-vanishing quark vev $\left\langle q_{4} \cdot \tilde{q}^{\tilde{4}}\right\rangle$, which completely breaks the gauge symmetry. The quark components that remain massless are identified with electric baryons. The superpotential arises here as the sum of dynamical and tree-level contributions, just as in the case considered above. This flow does not proceed through intermediate descriptions.

The oblique mass term $h Q^{3} \cdot Q^{4}$ in electric theory leads to more interesting flow of the second dual. It was considered in detail in ref. [12]. This flow is similar to that of the first dual under addition of the mass term $m \sqrt{\frac{-\Lambda^{2}}{\mu^{2}}} q^{4} \cdot \tilde{q}_{\tilde{4}}$. In the second dual, the electric baryons match to the magnetic ones as

$$
B^{i j} \simeq \sqrt{\frac{-\Lambda^{2}}{\mu^{2}}} \epsilon^{i j k l} C_{k l} \equiv \sqrt{\frac{-\Lambda^{2}}{\mu^{2}}} \epsilon^{i j k l} q_{k} \cdot q_{l},
$$

and the same for antibaryons. Thus, electric mass term $h Q^{3} \cdot Q^{4}$ corresponds to the term

$$
h \sqrt{\frac{-\Lambda^{2}}{\mu^{2}}} q_{1} \cdot q_{2} .
$$

For $h \gg \tilde{\Lambda}$, it gives mass to quarks $q_{1}$ and $q_{2}$. This term breaks $\mathrm{SU}(4)_{L}$ part of the flavour symmetry to diagonally embedded $\mathrm{SU}(2)_{12} \times \mathrm{SU}(2)_{34}$. The full superpotential now reads

$$
W=\frac{1}{\mu} M_{\tilde{i}}^{i} q_{i} \cdot \tilde{q}^{\tilde{j}}+h \sqrt{\frac{-\Lambda^{2}}{\mu^{2}}} q_{1} \cdot q_{2} .
$$

Integrating out massive quarks one obtains SQCD with $N_{f}=3$ quark flavours, mesons $M_{\tilde{j}}^{i}$ and tree-level superpotential

$$
W_{\text {tree }}=\frac{1}{\mu} M_{\tilde{i}}^{f} q_{f} \cdot \tilde{q}^{\tilde{j}}-\frac{1}{2 h \mu^{2}} \sqrt{\frac{\mu^{2}}{-\Lambda^{2}}} \epsilon_{a b} M_{\tilde{i}}^{a} M_{\tilde{j}}^{b} \tilde{\tilde{q}} \tilde{i}^{\tilde{i}} \cdot \tilde{q}^{\tilde{j}},
$$

where $a, b=1,2$ and $f, g=3,4$ are the indices of the representations of $\mathrm{SU}(2)_{12}$ and $\mathrm{SU}(2)_{34}$, respectively. At this stage the theory has global symmetry

$$
\mathrm{SU}(2)_{12} \times \mathrm{SU}(2)_{34} \times \mathrm{SU}(4)_{R} \times \mathrm{U}(1)_{B}
$$

where $\mathrm{U}(1)_{B}$ is a combination of the initial baryon charge and the third Cartan generator of $\mathrm{SU}(4)_{L}$ that does not belong to $\mathrm{SU}(2)_{12}$ or $\mathrm{SU}(2)_{34}$. In comparison to the electric 
$N_{f}=3 \mathrm{SQCD}$, this theory has extra $\mathrm{SU}(2)_{12}$ symmetry. This symmetry is the magnetic counterpart of electric $\mathrm{SU}(2)$ that acts on quarks $Q^{3}$ and $Q^{4}$ and is preserved by the oblique mass term $h Q^{3} \cdot Q^{4}$. This electric $\mathrm{SU}(2)$ completely disappears after the heavy quarks are integrated out. As opposed to electric theory, the intermediate magnetic theory contains mesons that transform non-trivially under $\mathrm{SU}(2)_{12}$. Hence, $\mathrm{SU}(2)_{12}$ is a non-trivial part of global symmetry of the second dual theory at this stage of its flow.

SQCD sector of this theory has $N_{f}=N_{c}+1$ flavors, and thus confines. The additional dynamical superpotential for its own composite mesons $N_{f}^{\tilde{i}}$ and baryons $C_{f g}, \tilde{C}^{\tilde{i}}$ is generated. The baryon fields $C_{f g}, \tilde{C}^{\tilde{i} \tilde{j}}$ can be replaced by $B^{a b}, \tilde{B}_{\tilde{i} \tilde{j}}$ by making use of the field redefinition (4.9). In this way we obtain the theory of baryons $B^{a b}$, antibaryons $\tilde{B}_{\tilde{i} \tilde{j}}$ and mesons $M_{\tilde{i}}^{a}, M_{\tilde{i}}^{f}, N_{f}^{\tilde{i}}$ with superpotential

$$
\begin{aligned}
W_{\mathrm{dyn}}=\frac{1}{4 h \Lambda^{2}}\left\{M_{\tilde{i}}^{a} M_{\tilde{j}}^{b}-\frac{1}{4} B^{a b} \tilde{B}_{\tilde{i} \tilde{j}}\right\} \epsilon^{\tilde{i} \tilde{j} \tilde{k} \tilde{l}} \epsilon_{a b} \tilde{B}_{\tilde{k} \tilde{l}}+ & \\
& \frac{1}{\mu} M_{\tilde{i}}^{f} N_{f}^{\tilde{i}}+\frac{1}{2 h \mu^{2}} \epsilon^{f g} N_{f}^{\tilde{i}} N_{g}^{\tilde{j}} \tilde{B}_{\tilde{i} \tilde{j}} .
\end{aligned}
$$

This superpotential gives masses to all fields that are absent in the electric low energy description, namely, to $M_{\tilde{i}}^{f}$ and $N_{f}^{\tilde{i}}$. After integrating them out, the remaining fields fit into $\mathrm{SU}(6)$ antisymmetric tensor representation, and the theory is equivalent to the common low energy description we obtained in previous cases.

For completeness, let us describe the flow of the third dual description [15]. It possesses full SU(8) flavour symmetry. Thus, any electric mass term has the same effect in the dual theory. It is easier to consider the flow of this theory in $\mathrm{SU}(8)$ notations. The matter fields are magnetic quarks $q_{I}, I=1, \ldots 8$, and elementary gauge singlet antisymmetric tensor $V^{I J}$ that contains all mesons and baryons. The latter corresponds to the moduli of electric theory. Superpotential of this dual takes the form

$$
W=\frac{1}{\mu} V^{I J} q_{I} \cdot q_{J} .
$$

Modulo SU(8) transformation, the magnetic counterpart of any electric mass term is $m V^{12}$. With this term in superpotential, quarks $q_{1}$ and $q_{2}$ obtain vev $\left\langle q_{1} \cdot q_{2}\right\rangle=-\mu m$ that breaks the gauge group. Then all quarks and the fields $V^{1 I}$ and $V^{2 I}$ become massive. Integrating them out one is left with the remaining components of $V^{I J}$ which transform as antisymmetric tensor under the surviving SU(6) flavour group, and no tree-level superpotential. Superpotential (4.1) is generated by instantons of the broken gauge group as described in $[11]$.

Hence, once mass term of any form is added, every theory given in table 1 flows to one and the same theory of SU(6) antisymmetric tensor field with superpotential given by eq. (4.1). This flow, however, is different for different duals and for different forms of the mass term.

\subsection{Making contact with index matching}

Finally, let us make contact with dualities for $N_{f}=3$ SQCD suggested by SV using the reduction of superconformal index identities of $N_{f}=4$. The conjecture is that there exist 
36 dual gauge theories, apart from the low energy description in terms of the antisymmetric $\mathrm{SU}(6)$ tensor. All of them are $\mathrm{SU}(2) \mathrm{SQCD}$ with $N_{f}=3$ flavours of quarks and additional singlet fields. Apart from the obvious electric $N_{f}=3 \mathrm{SQCD}$, there are two options for the field content and global symmetry. All other theories are different only in the sense explained in the previous section.

In notations of ref. [5], one of these two conjectured duals possesses $\mathrm{SU}(3)_{L} \times \mathrm{SU}(3)_{R} \times$ $\mathrm{U}(1)_{B} \times \mathrm{U}(1)_{\text {add }}$ global symmetry and besides SQCD sector contains 12 gauge singlet fields: $M_{1}$ in $\mathbf{3}_{\mathbf{A}}$ and $N_{1}$ in $\mathbf{3}$ of $\mathrm{SU}(3)_{L}$, and $M_{2}$ in $\mathbf{3}_{\mathbf{A}}$ and $N_{2}$ in $\mathbf{3}$ of SU(3) $R$, see table 12 of [5]. This theory is nothing but the theory obtained as an intermediate step in the flow of the first dual. In our notations, $M_{1}, N_{1}$ are baryons $B^{r s}, B^{r 4}$, and $M_{2}, N_{2}$ are antibaryons $\tilde{B}_{\tilde{r} \tilde{s}}, \tilde{B}_{\tilde{r} \tilde{4}}$. The group $\mathrm{U}(1)_{\text {add }}$ is $\mathrm{U}(1)_{4}$ entering (4.6). In addition, we have found that the superpotential of this theory is given by eq. (4.4).

Like its progenitor, the first dual for $N_{f}=4 \mathrm{SQCD}$, this theory has lower flavour group $\mathrm{SU}(3) \times \mathrm{SU}(3) \times \mathrm{U}(1)_{B} \times \mathrm{U}(1)_{4}$ in comparison with $\mathrm{SU}(6)$ of the electric $N_{f}=3$ SQCD. Thus, in the same way as in the previous section, multiple dualities emerge in this theory when global currents are coupled to external field. In this case, there are 20 duals that correspond to $C_{6}^{3}$ ways to split six eigenvalues of the Cartan generator of the $\mathrm{SU}(6)$ global symmetry into those acting on left and right magnetic quarks. We note here that unlike in $N_{f}=4$ case, interchanging left and right quarks in the magnetic theory does not leave that theory intact, as it changes the sign of the $\mathrm{U}(1)_{4}$ charge of baryons $B^{r 4}$; hence the above counting.

The second dual proposed by $\mathrm{SV}$ possesses $\mathrm{SU}(2)_{L} \times \mathrm{SU}(2)_{\text {add }} \times \mathrm{SU}(4)_{R} \times \mathrm{U}(1)_{B}$ global symmetry with 16 gauge singlet fields: $M$ in $(\mathbf{1}, \mathbf{2}, \mathbf{4})$ representation and $N$ in $(\mathbf{2}, \mathbf{1}, \mathbf{4})$ representation (cf. table 13 of $[5])$. We identify this theory with the intermediate description obtained during the flow of the second $N_{f}=4$ dual theory with $M$ identified with the mesons $M_{\tilde{i}}^{f}$ and $N$ with the mesons $M_{\tilde{i}}^{a}$, see (4.11). Its tree-level superpotential is given by (4.10).

By coupling global currents of this theory to external field one obtains 15 dualities, in the sense of the previous section. These correspond to $C_{6}^{2}$ ways to label six eigenvalues of the Cartan generator of SU(6) as two left and four right.

As a result, the total number of dualities (in external field sense) is $1+20+15=36$, taking into account electric $N_{f}=3 \mathrm{SQCD}$. Without the external field, there are two new dual descriptions for $N_{c}=2, N_{f}=3 \mathrm{SQCD}$ conjectured from index matching and supported by our study. Both are valid Seiberg dual theories, which show identical infrared behaviour. These descend from $N_{c}=2, N_{f}=4$ SQCD dualities by integrating out one flavour, and are $N_{c}=2, N_{f}=3 \mathrm{SQCD}$ with additional singlets. Tree level superpotentials in the two theories are given by (4.4) and (4.10), respectively. Unlike in many other cases, one can explicitly follow the flow of all three theories to the infrared and check that they have one and the same low energy description.

\section{Acknowledgments}

The author is indebted to S. Demidov, D. Gorbunov, V. Spiridonov, V. Vartanov and especially to V. Rubakov for useful discussions. The work has been supported in part 
by the Russian Foundation for Basic Research grant 08-02-00473a, by the grants of the President of the Russian Federation MK-4317.2009.2, NS-1616.2008.2 (government contract 02.740.11.0244), by FAE program (government contract P520), by the 'MassTeV' ERC advanced grant 226371, and by the 'Dynasty' foundation.

Open Access. This article is distributed under the terms of the Creative Commons Attribution Noncommercial License which permits any noncommercial use, distribution, and reproduction in any medium, provided the original author(s) and source are credited.

\section{References}

[1] C. Romelsberger, Calculating the superconformal index and Seiberg duality, arXiv:0707.3702 [SPIRES].

[2] J. Kinney, J.M. Maldacena, S. Minwalla and S. Raju, An index for 4 dimensional super conformal theories, Commun. Math. Phys. 275 (2007) 209 [hep-th/0510251] [SPIRES].

[3] C. Romelsberger, Counting chiral primaries in $N=1, D=4$ superconformal field theories, Nucl. Phys. B 747 (2006) 329 [hep-th/0510060] [SPIRES].

[4] F.A. Dolan and H. Osborn, Applications of the superconformal index for protected operators and q-hypergeometric identities to $N=1$ dual theories, Nucl. Phys. B 818 (2009) 137 [arXiv:0801.4947] [SPIRES].

[5] V.P. Spiridonov and G.S. Vartanov, Superconformal indices for $\mathcal{N}=1$ theories with multiple duals, Nucl. Phys. B 824 (2010) 192 [arXiv:0811.1909] [SPIRES].

[6] V.P. Spiridonov, Theta hypergeometric integrals, Algebra i Analiz 15 (6) (2003) 161 [St. Petersburg Math. J. 15 (2003) 929] [math/0303205].

[7] V.P. Spiridonov, Essays on the theory of elliptic hypergeometric functions, Uspekhi Mat. Nauk 63 (2008) 3 [Russian Math. Surveys 63 (2008) 405] [arXiv:0805.3135].

[8] E.M. Rains, Transformations of elliptic hypergometric integrals, to be published in Ann. of Math., math/0309252.

[9] E.M. Rains, $B C_{n}$-symmetric abelian functions, Duke Math. J. 135 (2006) 99 [math/0402113].

[10] V.P. Spiridonov and G.S. Vartanov, Elliptic hypergeometry of supersymmetric dualities, arXiv:0910.5944 [SPIRES].

[11] N. Seiberg, Exact results on the space of vacua of four-dimensional SUSY gauge theories, Phys. Rev. D 49 (1994) 6857 [hep-th/9402044] [SPIRES].

[12] R.G. Leigh and M.J. Strassler, Accidental symmetries and $N=1$ duality in supersymmetric gauge theory, Nucl. Phys. B 496 (1997) 132 [hep-th/9611020] [SPIRES].

[13] N. Seiberg, Electric-magnetic duality in supersymmetric non-abelian gauge theories, Nucl. Phys. B 435 (1995) 129 [hep-th/9411149] [SPIRES].

[14] C. Csáki, M. Schmaltz, W. Skiba and J. Terning, Self-dual $N=1$ SUSY gauge theories, Phys. Rev. D 56 (1997) 1228 [hep-th/9701191] [SPIRES].

[15] K.A. Intriligator and P. Pouliot, Exact superpotentials, quantum vacua and duality in supersymmetric $S P(N(c))$ gauge theories, Phys. Lett. B 353 (1995) 471 [hep-th/9505006] [SPIRES].

[16] G. 't Hooft, Naturalness, chiral symmetry, and spontaneous chiral symmetry breaking, NATO Adv. Study Inst. Ser. B Phys. 59 (1980) 135 [SPIRES]. 